\title{
Exclusive dijet production in diffractive deep inelastic scattering at HERA
}

\section{Marcin GUZIK*}

Akademia Górniczo-Hutnicza im. Stanistawa Staszica w Krakowie

E-mail: marcin.guzikedesy.de

\begin{abstract}
The exclusive production of dijets in diffractive deep inelastic lepton-proton scattering has been measured with the ZEUS detector at HERA using an integrated luminosity of $372 \mathrm{pb}^{-1}$ of $e^{ \pm} p$ data. The measurement has been performed in the kinematic range $Q^{2}>25 \mathrm{GeV}^{2}, 90<W<$ $250 \mathrm{GeV}$ and $x_{I P}<0.01$, where $Q^{2}$ is the virtuality of exchanged photon, $\mathrm{W}$ is the $\gamma^{*}-p$ centre of mass energy and $x_{I P}$ is the fraction of the proton momentum taken by the diffractive exchange. Jets have been reconstructed in the photon-Pomeron $\left(\gamma^{*}-I P\right)$ centre of mass frame using the exclusive $k_{T}$ algorithm. The cross section for the exclusive production of dijets is given as a function of the angle between the plane defined by exchanged photon and dijets momenta and the plane defined by the incoming and scattered lepton momenta in the $\gamma^{*}-I P$ rest frame. It is compared to theoretical predictions of models based on boson-gluon fusion and two-gluon exchange processes.
\end{abstract}

XXII. International Workshop on Deep-Inelastic Scattering and Related Subjects 28 April - 2 May 2014

Warsaw, Poland

\footnotetext{
*Speaker.

${ }^{\dagger}$ On behalf of the ZEUS Collaboration
} 


\section{Introduction}

Data used in this analysis were measured by the ZEUS detector that operated at DESY, Hamburg. They were collected during HERA II period, that is the period between years 2003 and 2007, when electrons or positrons accelerated to the energy of $27.5 \mathrm{GeV}$ were collided with $920 \mathrm{GeV}$ protons giving $318 \mathrm{GeV}$ of the centre of mass energy at the interaction point. Total luminosity of this data was equal to $372 \mathrm{pb}^{-1}$.

Denoting 4-momenta of incoming and scattered proton by $\mathbf{P}$ and $\mathbf{P}^{\prime}$, 4-momenta of incoming and scattered lepton by $\mathbf{k}, \mathbf{k}^{\prime}$ and defining 4-momentum of virtual photon $\gamma^{*}$ as $\mathbf{q}:=\mathbf{k}-\mathbf{k}^{\prime}$, standard kinematic variables describing deep inelastic scattering in $e^{ \pm} p$ collisions, such as virtuality of exchanged photon $Q^{2}:=-\mathbf{q}^{2}$, photon-proton centre of mass energy squared $W^{2}:=(\mathbf{P}+\mathbf{q})^{2}$, proton-lepton centre of mass energy squared $s:=(\mathbf{P}+\mathbf{k})^{2}$, Bjorken scaling variable $x=Q^{2} / 2 \mathbf{P} \cdot \mathbf{q}$, and also those related to diffraction, such as $x_{I P}=\left(\mathbf{P}-\mathbf{P}^{\prime}\right) \cdot \mathbf{q} / \mathbf{P} \cdot \mathbf{q}$ and $\beta=x / x_{I P}$ can be introduced.

\section{The aim of the analysis and definition of angle $\phi$}

There are two main mechanisms of production of diffractive dijets, that is 2-gluon exchange mechanism 1(a) and boson-gluon fusion mechanism 1(b). First of them (2-gluon exchange) is based on fully perturbative calculations for which only proton parton density function is needed. The latter one depends on partonic structure of the Pomeron and requires, diffractive parton density functions (dPDFs).

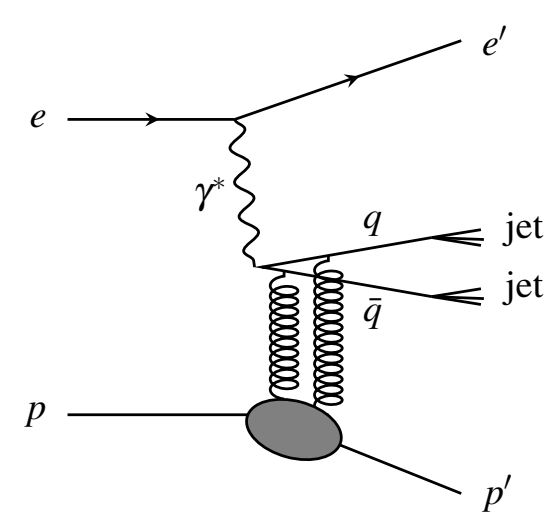

(a) 2-gluon exchange

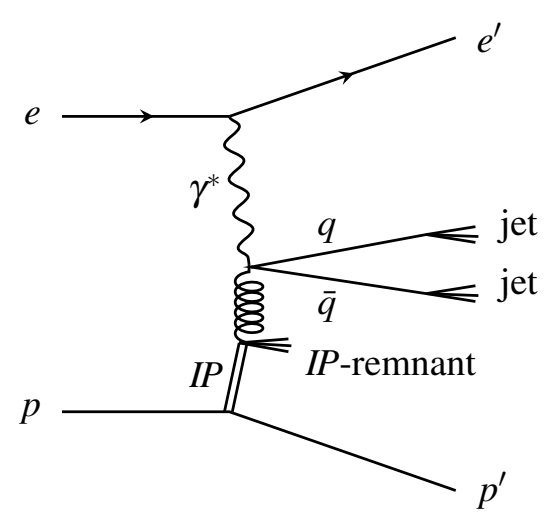

(b) Boson-Gluon Fusion

Figure 1: Diagrams of production of diffractive dijets.

This analysis was done in $\gamma^{*}$-IP centre of mass frame with $z$ axis along virtual photon momentum and $y$ axis direction defined by the cross product of virtual photon and scattered lepton momenta. The main aim of this analysis is the measurement of the angle $\phi$ between lepton plane, that is the plane spanned by incoming and scattered lepton momenta and jet plane, that is plane spanned by jets, which are in $\gamma^{*}$-IP centre of mass frame back-to-back and virtual photon itself. 


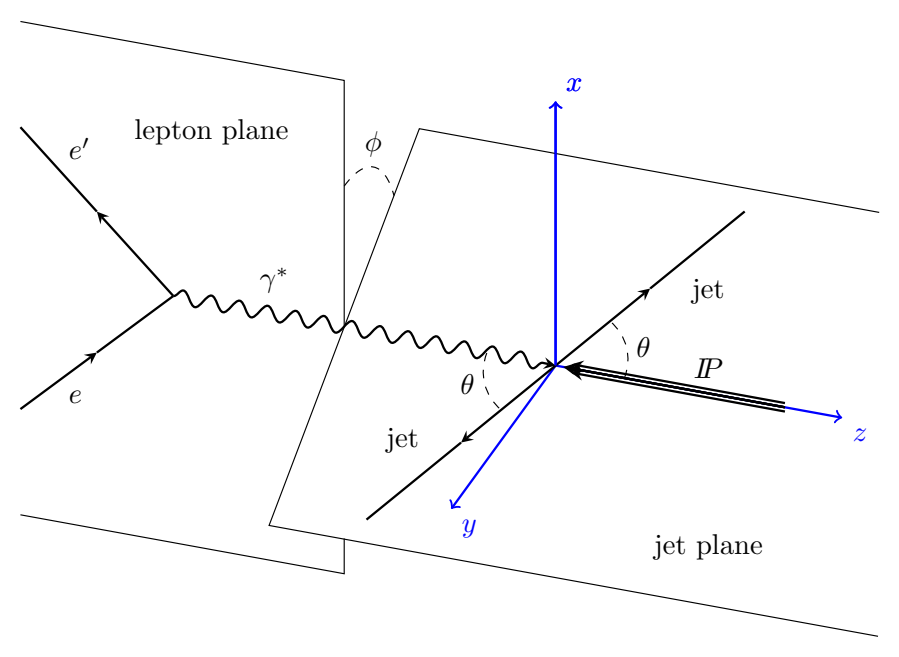

Figure 2: Virtual photon - pomeron centre of mass frame.

Thanks to the above definition of the coordinates this angle corresponds to the minimum of absolute values of azimuthal angles of jets. This azimuthal angular distribution is of particular importance because two aforementioned models that is 2-gluon exchange and BGF gives different prediction for the shape of single differential cross section with respect to $\phi$. Both predict that it is proportional to $(1+A \cos 2 \phi)$ but each of them gives different sign of the shape parameter $A$. In BGF $A$ should be positive whereas 2-gluon exchange model predicts it to be negative. So in principle to measure the shape of this distribution means to distinguish between those two models or at least to be able to tell which one dominates in nature.

\section{Monte Carlo Samples}

All Monte Carlo samples used during this analysis were generated with RAPGAP 3.01/26 [4], with hadronisation modelled by JETSET 7.4 [9] program which is based on the Lund string model [10]. Signal MC, used for unfolding the data to the hadron level, was generated with SATRAP [5, 6], that is colour dipole model with saturation as implemented in RapGap 3.01/26 with $q \bar{q}$ and $q \bar{q} g$ in a final state, and with radiation simulated with HERACLES 4.6.3. [11] This Monte Carlo was reweighted on the hadron level to improve description of the data.

Two additional smaller MC samples were generated as a hadron level predictions that is

- 2-gluon exchange model - RapGap 3.01/26

- BGF (resolved Pomeron) - RapGap 3.01/26.

Jets were found in $\gamma^{*}-I P$ centre of mass using the Durham Exclusive $k_{t}$ Jet Algorithm [7], with a jet resolution parameter $y_{\text {cut }}=0.15$, implemented in FastJet [8] package. 


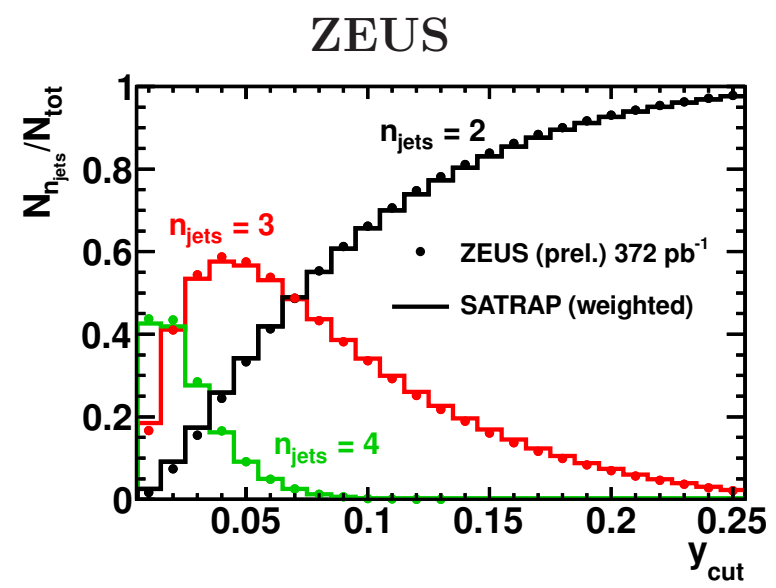

Figure 3: The ratios of events for which two, three or four jets were found to the total number of events in the final state as a function of the value of the resolution parameter, $y_{c u t}$. Data are shown as full dots. Monte Carlo is shown as histograms. Jets were reconstructed with $y_{c u t}=0.15$.

The figure 3 shows reasonable agreement between data and reweighted SATRAP Monte Carlo in distribution of number of jets as a function of $y_{\text {cut }}$.

\section{Transverse Energy Flows - The Shape of Exclusive Dijets in DIS}

\section{ZEUS}
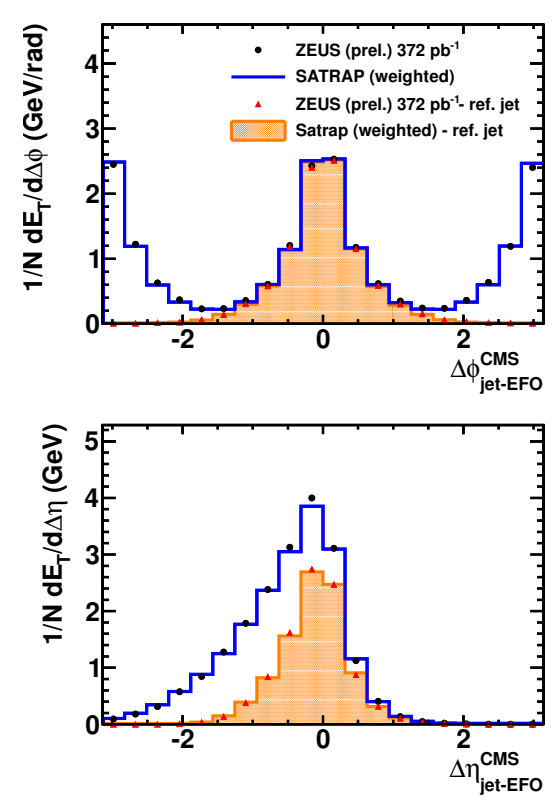

ZEUS
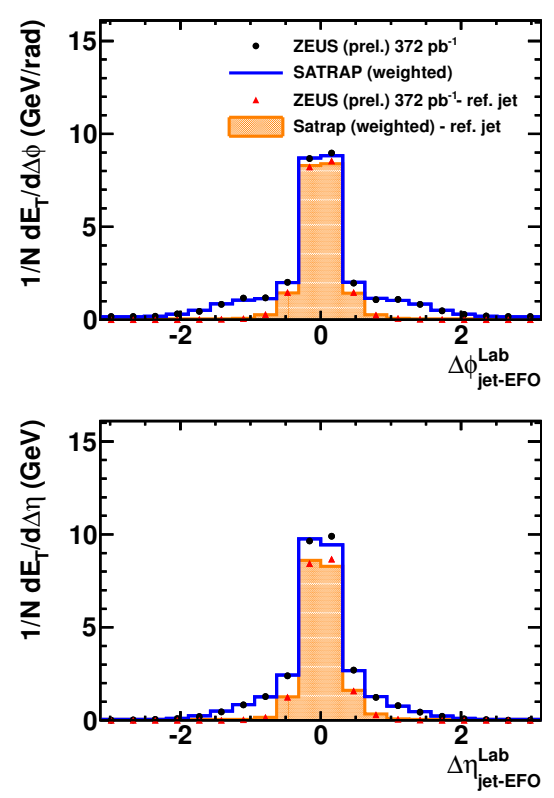

Figure 4: The transverse energy flows as functions of $\Delta \phi$ (top row) and $\Delta \eta$ (bottom row) in the $\gamma^{*}$-IP centre of mass frame (left column) and laboratory frame (right column). The reference jet is the jet with higher $p_{T}$ in the laboratory frame. Data are represented by full dots, the contribution from the reference jet in data by red triangles. Error bars represent statistical uncertainties. The reweighted SATRAP Monte Carlo is shown as histogram, blue line stands for full MC and orange-filled area contribution from the reference jet in MC. 
This analysis gives the chance not only to say something about the mechanism of production of dijets in diffractive DIS but also to examine the structure of exclusive jets themselves. This is why energy flows in $\gamma^{*}$-IP centre of mass frame and laboratory frame were measured. Results are showing that weighted SATRAP Monte Carlo describes the jet shape of exclusive dijet sample in both centre of mass and laboratory frames (cf. figure 4).

\section{Results}

The data were unfolded to the hadron level using TSVDunfold package [3], that is standard Singular Value Decomposition Unfolding with Regularisation implemented in ROOT. The normalised differential cross section $\frac{1}{\sigma} \frac{d \sigma}{d \phi}$ has been measured in the kinematic range presented below

$$
\begin{aligned}
90 \mathrm{GeV}<W & <250 \mathrm{GeV}, \\
25 \mathrm{GeV}^{2}<Q^{2} & , \\
x_{I P} & <0.01, \\
0.5<\quad & <0.7, \\
n_{\text {jets }} & =2, \\
2 \mathrm{GeV}< & p_{T \text { jet }} .
\end{aligned}
$$

The resulting distribution is presented in figure 5 .

\section{ZEUS}

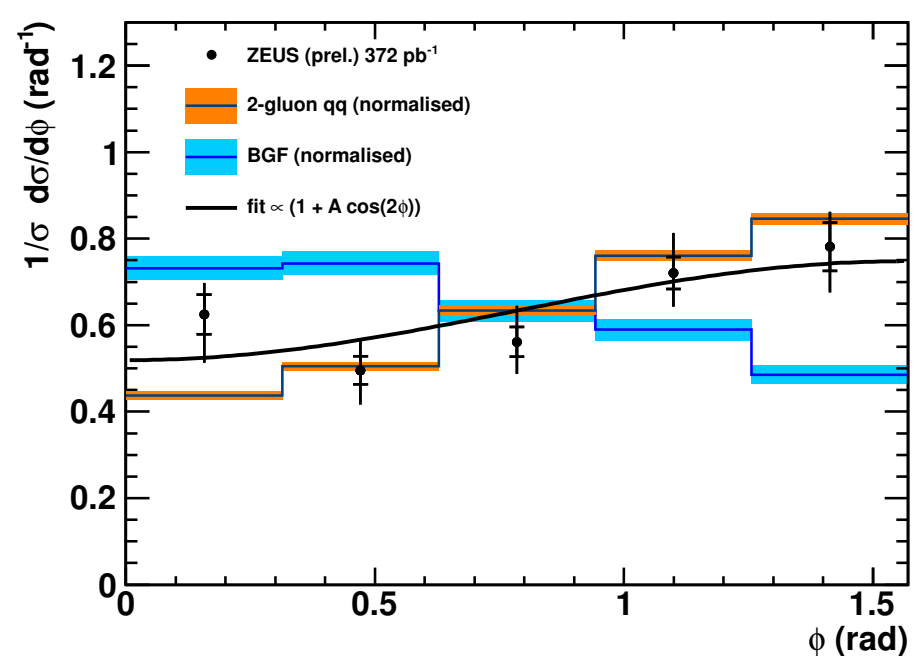

Figure 5: The normalised differential cross section $\frac{1}{\sigma} \frac{d \sigma}{d \phi}$ for exclusive diffractive production of dijets $e+p \rightarrow e+$ jet $_{1}+$ jet $_{2}+p$. The inner error bars indicate the statistical errors, the outer bars the statistical and systematic uncertainties. The black line represents the result of the fit to the function $\propto(1+A \cos 2 \phi)$ taking into account statistical errors only. The data is compared with distribution of Monte Carlo generated with resolved-Pomeron model [1] and the two-gluon exchange model [2] in which exclusive dijets originate from $q \bar{q}$ pairs only. Bands on the Monte Carlo distributions show only statistical uncertainties. Both predictions were normalised to one. 
Values of shape parameters $A$ fitted to the data and hadron level Monte Carlo samples are presented in the table below.

\begin{tabular}{lcc} 
& $A$ & $\chi^{2} / \mathrm{NDF}$ \\
\hline fit & $-0.18 \pm 0.06$ (stat. $)_{-0.09}^{+0.06}$ (sys.) & $4.11 / 3$ \\
\hline 2-gluon(qq) MC & $-0.34 \pm 0.01$ (stat.) & \\
BGF MC & $0.21 \pm 0.02$ (stat.) & \\
\hline
\end{tabular}

To summarise, the shape of $\phi$ distribution of exclusive dijets in diffractive DIS has been measured for the first time at HERA. The data favour 2-gluon exchange model over boson-gluon fusion giving negative shape parameter $A$ within two sigma range.

\section{Acknowledgements}

This work was partly supported by the National Science Centre under contract No. DEC-2012/06/M/ST2/00428.

\section{References}

[1] G. Ingelman and P. Schlein, "Jet Structure in High Mass Diffractive Scattering," Phys.Lett. B152 (1985) 256.

[2] J. Bartels, H. Lotter, and M. Wusthoff, "Quark - anti-quark production in DIS diffractive dissociation," Phys.Lett. B379 (1996) 239-248.

[3] Nucl. Instrum. Meth. A372 (1996) 469-481

[4] H. Jung, The Rapgap Monte Carlo for Deep Inelastic Scattering, 3.1 ed. http://rapgap.hepforge.org/.

[5] K. J. Golec-Biernat and M. Wusthoff, "Saturation in diffractive deep inelastic scattering," Phys.Rev. D60 (1999) 114023.

[6] K. J. Golec-Biernat and M. Wusthoff, "Saturation effects in deep inelastic scattering at low $Q^{2}$ and its implications on diffraction," Phys.Rev. D59 (1998) 014017.

[7] S. Catani, Y. L. Dokshitzer, M. Olsson, G. Turnock and B. R. Webber, Phys. Lett. B 269 (1991) 432

[8] M. Cacciari, G.P. Salam and G. Soyez, Eur.Phys.J. C72 (2012) 1896

[9] T. Sjostrand, "High-energy physics event generation with PYTHIA 5.7 and JETSET 7.4." Comput.Phys.Commun. 82 (1994), 74.

[10] B. Andersson, G. Gustafson, G. Ingelman and T. Sjostrand, "Parton Fragmentation and String Dynamics." Phys.Rept. 97 (1983), 31.

[11] A. Kwiatkowski, H. Spiesberger and H.J. Mohring, "Heracles: An Event Generator for ep Interactions at HERA Energies Including Radiative Processes: Version 1.0." Comput.Phys.Commun. 69 (1992), 155. 